EPJ Web of Conferences 64, 03001 (2014)

DOI: $10.1051 /$ epjconf/ 20146403001

(C) Owned by the authors, published by EDP Sciences, 2014

\title{
Accretion on to Magnetic White Dwarfs
}

\author{
Dayal Wickramasinghe ${ }^{1}$ \\ ${ }^{1}$ Australian National University, Canberra ACT
}

\begin{abstract}
The Magnetic Cataclysmic Variables (MCVs) are close interacting binaries where mass is transferred from a late type secondary star to a magnetic white dwarf. Two modes of accretion can be identified depending on the strength of the magnetic field, the mass transfer rate and orbital parameters. (a) Disced Accretion: In the Intermediate polars (IPs), the mass transfer stream circularises and forms an accretion disc. Material couples on to field lines in a narrow inner transition region where the velocity in the orbital plane changes from Keplerian to co-rotation (b) Discless Accretion: In the polars, the accretion stream is disrupted by the magnetic field before it can circularise. Material couples on to field lines via an inner transition region where the velocity changes from essentially free fall to co-rotation.

The polars have no counterparts in neutron star systems and their study provides unique insights into the complex nature of the magnetospheric boundary. The observed properties of accretion shocks at the white dwarf surface such as the anomalous soft-X-ray excess and its time variability provide strong support for the hypothesis that under certain circumstances the field channelled funnel flow is "blobby". This has been attributed to interchange instabilities such as the Magnetic Rayleigh-Taylor instability in the shocked gas at the stream-magnetosphere boundary where the stream fragments into discrete clumps of gas. As the clumps penetrate into the magnetosphere, they are shredded into smaller mass blobs via the Kelvin-Helmholtz instability that then couple on to field lines over an extended inner transition region in the orbital plane. The more massive blobs penetrate deep into the photosphere of the white dwarf releasing their energy as a reprocessed soft$\mathrm{X}$-ray black body component. Although similar instabilities are expected in the inner transition region in disced accretion albeit on a different scale there has been no direct observational evidence for blobby accretion in the generally lower field and disced IPs.
\end{abstract}

\section{Introduction}

Cataclysmic variables (CVs) are close interacting binaries that contain a white dwarf as the primary star. The orbital periods are in the range $P_{\text {orb }} \sim 80 \mathrm{~min}-10 \mathrm{hrs}$ and the mass transferring secondary is typically a late type main sequence star [31]. Some $25 \%$ of CVs have white dwarfs with strong surface magnetic fields $\left(B \sim 10^{6}-10^{9} \mathrm{G}\right)$ and are known as the magnetic cataclysmic variables (MCVs). The field strengths are similar to those found in the isolated high field magnetic white dwarfs (HFMWDs) which comprise some $10-15 \%$ of all white dwarfs. The fields are several orders of magnitude stronger $\left(\sim 10^{3} \mathrm{G}\right)$ than what may be expected for dynamo generated fields in the convective cores of intermediate mass main sequence stars during single star evolution. The origin of the strong fields in magnetic white dwarfs has been debated over the decades. While the fossil field hypothesis has

This is an Open Access article distributed under the terms of the Creative Commons Attribution License 2.0, which permits unrestricted use, distribution, and reproduction in any medium, provided the original work is properly cited. 
been the favoured interpretation (see review [34]), it now appears more likely that the strong fields in both the isolated magnetic white dwarfs and the white dwarfs in MCVs are generated by a dynamo mechanism that feeds on the differential rotation that ensues during a common envelope phase of evolution. Systems that merge during this process form the isolated HFMWDs while those that just avoid a merge result in the MCVs ([28],[35]).

The magnetic nature of CVs is generally revealed by the detection of coherent pulsations in the optical and /or X-ray band attributed to the spin period of the magnetic white dwarf. The pulsations are attributed to the presence of one or more accretion hot spots at the base of magnetically confined accretion flows that channel material on to the white dwarf surface. The MCVs divide into two groups, the Polars and the Intermediate Polars (IPs). The polars, as their name implies, emit strongly circularly (and linearly) polarised radiation in the optical - near infra-red bands due to high harmonic electron cyclotron emission from accretion shocks. The spacing of the cyclotron harmonics gives a direct measure of the magnetic field strength at the shock/shocks and yield fields of $\sim 5 \times 10^{6}-10^{9} \mathrm{G}$ [29]. A characteristic of the polars is that the spin period of the white dwarf $P_{\mathrm{s}}$ equals the orbital period $P_{\text {orb }}$ so that the magnetic white dwarf is phase locked into synchronous rotation with the orbit and accretion occurs directly from the accretion stream without the mediation of an accretion disc. In contrast in the IPs $P_{\mathrm{s}}<P_{\text {orb }}$ and there is usually some evidence for a truncated disc. Polarised emission is rarely seen in the optical - near infra-red bands suggestive of generally lower fields.

There are a few MCVs with properties that are intermediate to those of polars and IPs. These are the polar like systems where the white dwarf is very close to spin equilibrium with the orbit $\left(\left|P_{\mathrm{s}} / P_{\text {orb }}\right| \simeq 10^{-2}\right)$, and IP like systems with $P_{\mathrm{s}}<P_{\text {orb }}$ but which do not show evidence for an accretion disc. The former are likely to be polars that have been driven out of synchronism due to a recent nova outburst. The latter may represent systems that exhibit a new type of spin equilibrium - namely spin equilibrium with an accretion stream. Alternatively, they may be systems that are evolving towards or away from a disced IP state [13].

In this paper we review the theory of accretion on to magnetic white dwarfs in MCVs. We show how the polars and the IPs allow us to investigate different aspects of the interaction of accretion flows with the magnetospheres of white dwarfs. We focus mainly on the polars and show how both theory and observations provide strong support for a "blobby" accretion model where interchange instabilities play a role in determining the properties of the field channelled flow.

\section{The Accretion flows in IPs and Polars}

The problem of Roche lobe overflow and disc formation in semi-detached binary was discussed by Lubow and Shu [22]. The accretion stream that emerges from the inner Lagrangian point $L_{1}$ first free falls towards the primary reaching a minimum distance $\varpi_{\min }$. It then reverses direction collides with itself and circularises at a radius $\varpi_{\max }$. A ring forms at the circularsation radius and then spreads to form a disc through the outward transport of angular momentum via viscous stresses.

The presence of a magnetic field in the primary star could interfere with the above process and lead to two main accretion modes depending on the orbital parameters and the strength and geometry of the field. The magnetic field may inhibit the formation of a disc with accretion occurring direct from the stream or a disc may form. If one makes the simplifying assumption that the magnetic field is dipolar in the region of interaction with the accretion flow various estimates can be made of the magnetospheric radius for these two modes of accretion. 


\subsection{Disc Accretion}

The magnetospheric radius $R_{\mu}^{\text {disc }}$ is defined as the radius at which magnetic stresses first become comparable to material stresses in the disc and affects the dynamics of the Keplerian disc. For accretion to proceed within this radius one requires the fastness parameter

$$
\omega_{\mathrm{s}}=\Omega_{\mathrm{s}} / \Omega_{K}\left(R_{\mu}^{\mathrm{disc}}\right)=\left(R_{\mu}^{\mathrm{disc}} / R_{\mathrm{c}}\right)^{3 / 2} \leq 1
$$

where $\Omega_{\mathrm{s}}$ is the angular velocity of the star, $R_{\mathrm{c}}=\left(G M_{1} / \Omega_{\mathrm{s}}^{2}\right)^{1 / 3}$ is the co-rotation radius and $M_{1}$ is the mass of the primary star. In most models this occurs over a narrow inner transition region of width $\delta R_{\text {coup }}=R_{\mu}^{\text {disc }}-R_{0}<<R_{\mu}^{\text {disc }}$ where $R_{0}$ is the true inner radius of the disc.

Estimates of $R_{\mu}^{\text {disc }}$ and $R_{0}$ depend in particular on assumptions made on the magnetic diffusivity of the disc and the role played by instabilities and reconnection in limiting the toroidal field produced by the vertical shear between the Keplerian disc and the co-rotating magnetosphere. The disc may fully exclude the field (the diamagnetic case) or the field may partially penetrate the disc. Arguing for a dissipative disc Ghosh and Lamb [11] give

$$
R_{\mu}^{\mathrm{disc}}=0.52 \phi R_{\mu}^{\mathrm{sph}}=6.3 \times 10^{10} \phi\left(B_{7}^{\mathrm{p}}\right)^{4 / 7} \dot{M}_{16}^{-2 / 7} \hat{M}_{1}^{-1 / 7}\left(R_{\mathrm{wd}, 9}\right)^{12 / 7} \mathrm{~cm}
$$

where $R_{\mu}^{\mathrm{sph}}$ is the magnetospheric radius for spherical accretion and $\phi$ is a parameter of the order of unity. Here $B_{7}^{\mathrm{p}}$ is the effective polar field strength for an assumed dipolar field structure in units of $10^{7} \mathrm{G}, \dot{M}_{16}$ is the mass transfer rate in units of $10^{16} \mathrm{gs}^{-1}, \hat{M}_{1}$ is the mass of the white dwarf in solar masses and $R_{\mathrm{wd}, 9}$ is its radius in units of $10^{9} \mathrm{~cm}$.

According to a recent study [5] the rapid increase of the magnetic torque over the viscous torque in the inner transition region is accompanied by additional heating due to magnetic dissipation and a consequent thickening of the disc. It is argued that the enhanced vertical pressure gradient assists in driving a supersonic sub-Alfvenic flow overcoming the gravitational-centrifugal potential barrier. These calculations which were for an aligned dipole suggests that under certain circumstances the initiation of a field channelled flow may require additional "thermal assistance" [5] over and above what is provided by viscous heating. The relevance of these considerations for the general case of accretion on to an inclined dipole will be discussed in section 3.2 in the context of polars.

The study of IPs where the shock is eclipsed by the companion star could in principle yield information on the geometry of the shock and hence through back projection on the inner transition region in the orbital plane in disced accretion. Such studies are, however, rare and have so far only provided limited information on the nature of this region [13].

\subsection{Stream Accretion}

In stream accretion the material that leaves the inner Lagrangian point forms a ballistic stream that free falls towards the magnetic white dwarf. Compared to the case of disc accretion where the material drifts slowly inwards with an inward radial velocity $v_{\mathrm{r}} \sim 10^{-2} v_{\mathrm{ff}}$ as it first encounters the field, in stream accretion, the material that first encounters the stream has velocities $\left(\sim v_{\mathrm{ff}}\right)$ that are typically $\sim 100$ times higher. It may therefore be more appropriate to consider the stream as diamagnetic. The stream then penetrates the magnetosphere excluding the magnetic field via screening currents until the ram pressure $\rho_{\mathrm{s}} v_{\mathrm{ff}}(r)^{2}$ equals the magnetic pressure $B^{2}(r) / 8 \pi$. From the continuity equation we also have $\pi d^{2} \rho_{\mathrm{s}} v_{\mathrm{ff}}(r)=\dot{M}$ where $d$ is the diameter of the stream. From [22] and the parametrized fits in [14] $d \simeq 10^{9} \eta P_{\text {orb }, 5} \mathrm{~cm}$ along the stream trajectory where $\eta$ is a factor of order unity. Combining these expressions we obtain a stream magnetospheric radius

$$
R_{\mu}^{s t}=1.75 \times 10^{10} \eta^{4 / 11}\left(B_{7}^{\mathrm{p}}\right)^{4 / 11} \dot{M}^{-2 / 11} P_{\mathrm{orb}, 5}^{4 / 11} \hat{M}_{1}^{-1 / 11}\left(R_{\mathrm{wd}, 9}\right)^{12 / 11} \mathrm{~cm}
$$




\subsection{IP or Polar?}

We consider the problem of the formation and persistence of accretion discs. The circularisation radius is given approximately by

$$
R_{\text {circ }} \simeq \varpi_{\max } \simeq 0.2 \xi R_{L} \simeq 10^{10} \xi P_{\mathrm{orb}, 5}^{2 / 3} \hat{M}_{1}^{1 / 3} \mathrm{~cm}
$$

where $R_{L}$ is the distance of the inner Lagrangian point from the white dwarf and $\xi$ is a parameter which is of the order unity. Typically $\varpi_{\max } / \varpi_{\min } \simeq 2$ over a wide range of orbital periods [22]. To focus our discussion we scale our estimates to systems with an orbital period of $5 \mathrm{hr}$ where both IPs and polars are found and assume typical accretion rates of $10^{16} \mathrm{gs}^{-1}$.

A disc will certainly form if the infalling accretion stream can turn around and circularise without interference by the magnetic field. A minimum requirement for disc formation is therefore $R_{\mu}^{\text {st }}<\varpi_{\min }$ or

$$
B_{p} \leq 3.2 \times 10^{5} \xi^{11 / 4} \eta^{-1} \dot{M}_{16}^{1 / 2} P_{\text {orb }, 5}^{5 / 6} \hat{M}_{1}^{7 / 6}\left(R_{\mathrm{wd}, 9}\right)^{-3} \mathrm{G}
$$

For a persistent disc at these orbital periods we require $R_{\mu}^{\text {disc }}<\varpi_{\max }$ - that is the specific angular momentum of the inner edge of the disc cannot exceed the specific angular momentum of the orbit at the circularisation radius. Interestingly, this condition also gives a maximum field $\sim 4 \times 10^{5} \mathrm{G}$ close to the above value.

After circularisation a steady state accretion disc will develop. The white dwarf will be in spin equilibrium with a truncated disc (an IP) or if $R_{\mu}^{\text {disc }} \leq R_{\mathrm{wd}}$ the disc will extend all the way to the white dwarf surface. The latter systems will have fields less than

$$
B_{p}=7 \times 10^{3} \phi^{-7 / 4} \dot{M}_{16}^{1 / 2} \hat{M}_{1}^{1 / 4}\left(R_{\mathrm{wd}, 9}\right)^{-5 / 4} \mathrm{G}
$$

and be classified as non magnetic CVs.

On the other hand if $R_{\mu}^{\text {st }}>\varpi_{\max }$ the stream would be diverted from its feee path before circularisation and a disc will not form in the normal way. In such systems accretion will occur directly from the accretion stream and spin equilibrium will be achieved not with a disc but through magnetic interactions with the companion star. The minimum field for such a Polar is therefore

$$
B_{p} \geq 2.1 \times 10^{6} \xi^{11 / 4} \eta^{-1} \dot{M}_{16}^{1 / 2} P_{\text {orb }, 5}^{5 / 6} \hat{M}_{1}^{7 / 6}\left(R_{\text {wd }, 9}\right)^{-3} \mathrm{G}
$$

These estimates are generally consistent with the observations of MCVs when allowance is made for uncertainties in the parameters $\eta$ and $\xi$ and the dependence on mass accretion rate and orbital period.

The fate of a system with $\varpi_{\min }<R_{\mu}^{\text {st }} \leq \varpi_{\max }$ is less clear. In such systems the magnetic field will prevent the stream from circularising in the manner envisaged in [22]. This has led some investigators to propose a new accretion mode where the magnetic white dwarf is in spin equilibrium with an accretion stream [15].

In CVs mass transfer is driven by angular momentum loss by stellar winds at long orbital periods and by gravitational radiation at short periods. The mean mass transfer rate generally decreases with orbital period and is also observed to effectively cease in the period gap $P_{\text {orb }}=2-3 \mathrm{hr}$. Some systems which are disced IP's at long orbital periods can therefore become discless polars at lower orbital periods with implications for the interpretation of the observations of MCVs ([18], [32]).

\subsection{Spin Equilibria}

In IPs, spin equilibrium is achieved by a balance between the net magnetic torque $G_{\text {mag }}$ that operates between the magnetic white dwarf and the disc and the accretion (or matter) torque $G_{\text {acc }}$. The estimation of the accretion torque has been the subject of some debate. Material stresses are transferred 


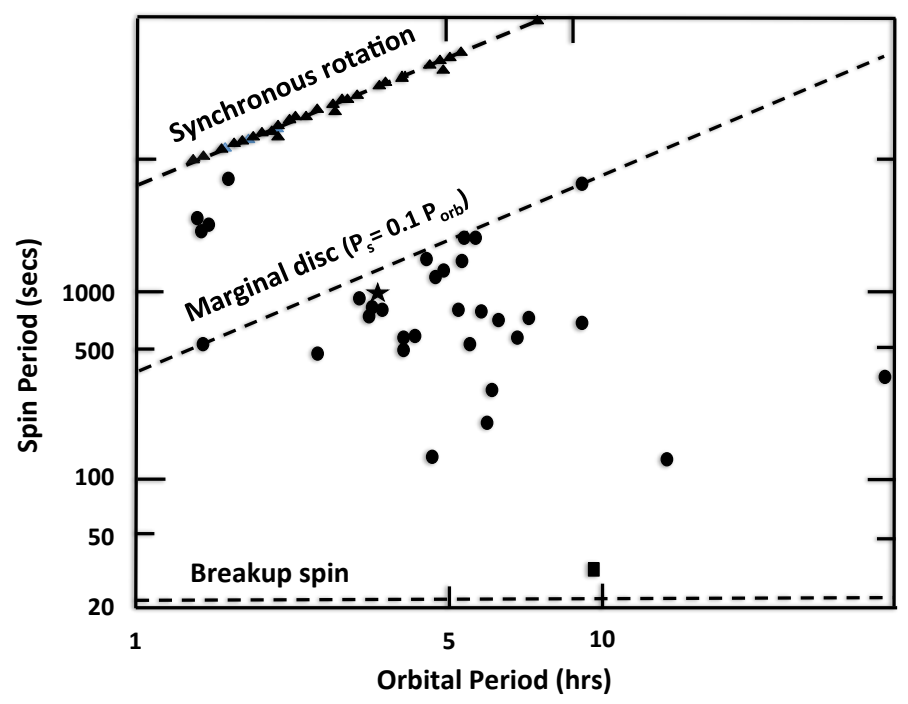

Figure 1. The Spin-Orbit Diagram for MCVs. The polars (filled triangles) hug the line corresponding to spin equilibrium with the orbit. The IPs (filled circles) with $P_{\text {orb }} \geq 3 \mathrm{hr}$ scatter about the line where the spin period is a tenth of the orbital period corresponding to spin equilibrium with a "marginal" disc as discussed in the text. The filled star is the "discless" IP V2400Oph. The filled square is AE Aquarii which is an IP with $P_{\mathrm{s}}=33 \mathrm{~s}$ in the propellor phase. The breakup spin line of a typical white dwarf is shown at the lower boundary of this diagram. Data based on compilation of IPs by Mukai in http://heawww.gsfc.nasa.gov/users/mukai/iphone/iphone.html.

to the white dwarf via the twist induced in the field lines by the field aligned flow. In the case of a perfectly conducting white dwarf atmosphere with a no slip boundary condition at its surface accretion can, in principle, proceed with zero matter torque with all of the matter angular momentum at the coupling radius transferred back into the disc [19] or into outflows. Under more realistic conditions one would expect $G_{\text {acc }}<\dot{M}\left[R_{\mu}^{\text {disc }}\right]^{2} \Omega_{K}\left(R_{\mu}^{\text {disc }}\right.$ [7]. The spin up and spin down magnetic torques that are exerted by the disc on the spinning magnetic white dwarf are also difficult to estimate since they require estimates of the azimuthal twist of the field lines that penetrate the disc which depend on uncertain assumptions of magnetic diffusivity and reconnection time scales in the disc. The equilibrium spin period is therefore usually expressed in parametrized form in terms of a critical (equilibrium) value for the fastness parameter defined by $R_{\mu}=\omega_{\mathrm{s}}^{* 3 / 2} R_{\mathrm{c}}$ as

$$
P_{\text {eq }}^{\text {disc }}=2.4 \times 10^{4}\left(0.35 / \omega_{\mathrm{s}}^{*}\right) \phi^{3 / 2}\left(B_{7}^{\mathrm{p}}\right)^{6 / 7} \dot{M}_{16}^{-3 / 7} \hat{M}_{1}^{-5 / 7} R_{\mathrm{wd}, 9}^{18 / 7} \mathrm{~s}
$$

Estimates of $\omega_{\mathrm{s}}^{*}$ range from $\sim 0.3$ to 1 depending on assumptions made (see e.g. [21]).

For accretion to proceed via a truncated disc the inner disc radius cannot exceed the circularisation radius. Treating the inner transition region as a narrow boundary layer, this requires $R_{\mu}=\omega_{\mathrm{s}}^{* 3 / 2} R_{\mathrm{c}} \leq$ 
$R_{\text {circ }}$. For IPs, the ratio of the equilibrium spin period to orbital period must therefore satisfy

$$
P_{\mathrm{eq}}^{\mathrm{disc}} / P_{\text {orb }} \leq 0.09\left(0.35 / \omega_{\mathrm{s}}^{*}\right)
$$

Polars exhibit spin - orbit coupling where the white dwarf is locked into spin equilibrium with the orbital period. Calculations have shown that when far from spin equilibrium, the white dwarf is spun towards equilibrium by dissipative torques that operate between the magnetic field of the white dwarf and the turbulent outer layers of the secondary. These torques bring the spin of the white dwarf close to spin equilibrium with the orbit [6]. The white dwarf is held in spin equilibrium by nondissipative magneto-static torques that operate between the magnetic white dwarf and the magnetic field (intrinsic) of the secondary star [4]. While the accretion torque $G_{\text {acc }}$ is again difficult to calculate, it is estimated to be much smaller than the magneto-static torques that keep the two stars locked in synchronous rotation so that a knowledge of its precise value is not as critical for understanding the overall properties of the polars. However, the direction of the torque and the asymmetries present in the white dwarf field structure (e.g. dipole, quadrupole or higher order multipole structures) both play a role in determining the favoured locked position. If we approximate the field by a dipole+quadrupole structure, or effectively by an offset dipole, we may expect the dipole axis to lead the line of centres with the weaker pole being closest to the orbital plane [33].

The clustering of IPs near the "critical" disc line $P_{\mathrm{s}} / P_{\text {orb }}=0.1$ in the spin-orbit diagram for orbital periods $P_{\text {orb }}>2 \mathrm{hr}$ was first noted by [32]. They also noted that IPs as a group tend to show period derivatives $|\dot{P} / P| \sim 10^{-6}-10^{-7}\left(\mathrm{yr}^{-1}\right)$ that are both positive and negative consistent with the idea that most systems are in near spin equilibrium with a disc with inner radii close to the circularisation radius. These ideas were subsequently further developed in [16].

We show in Figure 1 an updated spin-orbit diagram for polars and IPs. This diagram shows how the vast majority of the polars lie on the $P_{\mathrm{s}}=P_{\text {orb }}$ line exhibiting spin-orbit coupling. During a nova outburst the white dwarf could be driven out of synchronism but returns to synchronism of a time scale of $\sim 10^{3}-10^{4} \mathrm{yr}$ explaining the few systems that show small deviations from this line. The systems with $P_{\mathrm{s}} / P_{\text {orb }}<<0.1$ could generally be interpreted as lower field systems with more extensive truncated discs. The systems above this line are mostly systems that are out of spin equilibrium. We draw attention to the unique system AE Aqarii $\left(P_{\mathrm{s}}=33 \mathrm{~s}, P_{\text {orb }}=9.88 \mathrm{hr}\right)$ with a K4-K5 secondary star. This rapidly spinning magnetic white dwarf is currently spinning down and emission line observations show clear evidence for high velocity outflows indicative of a propellor phase [37].

The question of whether there could be accreting systems that are in spin equilibrium with a stream is an interesting one. If such systems exist they must be very rare since they should be showing evidence for the propelloring that would be required to balance the the accretion torque. For a more complete account of the observed properties of IPs see [13] and the review by Hellier given at this conference.

\section{Stream accretion in Polars}

\subsection{Entry into the magnetosphere}

The gas stream that leaves the inner Lagrangian point initially following a ballistic trajectory plunges into the combined magnetosphere of the phase locked stars with its entrapped wind [20] until the magnetic field influences its motion. On the diamagnetic approximation the stream excludes the field through the generation of surface currents until the ram pressure of the stream becomes comparable

with the magnetic pressure as assumed in our estimation of $R_{\mu}^{\text {st }}$. A shock will form at this radius, and if the shock is strong, the gas will be heated to a temperature

$$
T_{\mathrm{s}}^{\mathrm{st}}=3.7 \times 10^{7}\left(\hat{M}_{1} / R_{\mu, 10}^{\mathrm{st}}\right) K
$$


and the density will increase by a factor 4 to

$$
\rho_{2}=7 \times 10^{-11} \eta^{-2} \dot{M}_{16} P_{\text {orb }, 5}^{-2} \hat{M}_{1}^{-1 / 2}\left(R_{\mu, 10}^{\mathrm{st}}\right)^{1 / 2} \mathrm{~g} \mathrm{~cm}^{-2}
$$

The corresponding stand-off shock height for bremmstrahlung cooling is (e.g. [34])

$$
D_{\mathrm{s}}=2 \times 10^{9} \eta^{2} \dot{M}_{16}^{-1} P_{\mathrm{orb}, 5}^{2} \hat{M}_{1}^{3 / 2}\left(R_{\mu, 10}^{\mathrm{st}}\right)^{-3 / 2} \mathrm{~cm}
$$

In the post shock subsonic flow, the gas will cool from $4 \times 10^{7} \mathrm{~K}$ to $2 \times 10^{4} \mathrm{~K}$ with the density increasing to $\rho_{3} \sim 2000 \rho_{2}$ at the magnetospheric boundary [12]. It is envisaged that this material will enter the magnetosphere in the form of clumps through the development of various interchange instabilities such as the Rayleigh-Taylor (RT) instability as first discussed in the context of spherically symmetric accretion on to magnetised compact stars by Arons and Lea [1]. These authors assume that the scale of the structures that will become unstable will be set by the "lid size" $\left(\sim 0.1 D_{\mathrm{s}}\right)$ which is also the expected length scale of the cooled post-shock material.

The magnetic field can suppress or change the nature of the development of the instability depending on the strength and orientation of the field and set the scale of the instability. For face-on encounters where the wavenumber of the disturbance $\vec{k} \perp \vec{B}$ the field lines are simply pushed apart and the magnetic pressure compresses and elongates the clumps. Or the field could suppress the instability if $\vec{k} \| \vec{B}$ with the magnetic tension inhibiting its growth ([3],[30]). From linear theory, the strongest growing modes occur at twice the critical wavelength $\lambda_{0}=B^{2} \cos ^{2} \theta / g \Delta \rho$ below which the instability is suppressed. Here $B$ and $g$ are the field and acceleration due to gravity at the shock, $\Delta \rho$ is the density differential between the heavy and light fluid which we take to be $\rho_{3}$, and $\theta$ is the angle between $\vec{k}$ and $\vec{B}$. Scaling to parameters at the stream shock we estimate

$$
\lambda_{0}=5 \times 10^{8} \cos ^{2} \theta\left(B_{7}^{\mathrm{p}}\right)^{2}\left(R_{\mu, 10}^{\mathrm{st}}\right)^{-4.5} \dot{M}^{-1} P_{\mathrm{orb}, 5}^{2} \hat{M}_{1}^{-1.5} R_{\mathrm{wd}, 9}^{6} \mathrm{~cm}
$$

From (12), (13) and (3) we find $\lambda_{0} / D_{\mathrm{s}} \sim 5 \times 10^{-2}\left(B_{7}^{\mathrm{p}}\right)^{10 / 11} \dot{M}_{16}^{6 / 11} P_{\text {orb,5 }}^{-12 / 11}$ so that we may typically expect clumps of size $(.01-.1) D_{\mathrm{s}}$ to enter the magnetosphere in polars.

The fate of these clumps depend on the relative importance of two critical time scales: the dynamical time scale $t_{\mathrm{dyn}} \sim \Omega_{\mathrm{K}}^{-1}$ and the time scale $t_{\mathrm{diff}}$ for the diffusion of the field into the clump. The latter is expected to increase with the mass of the clump but difficult to estimate. Possible scenarios are

- The smaller mass clumps diffuse into field lines and are then channelled on to the surface of the white dwarf in the immediate vicinity of the post-shock region where the RT instability first develops.

- The larger mass clumps follow a dynamical trajectory under the action of gravity and the drag force exerted by the magnetic field. Material stripped off by the Kelvin-Helmholtz instability form smaller mass blobs that diffuse into field lines and are channelled on to the surface of the white dwarf [15].

- The blobs may already be channelled in-between field lines by magnetic forces even before the mass decreases to the point where the field diffuses into the blobs [1]

A proper understanding of the nature of the post-shock flow requires a full treatment of the MHD problem that allows for the development of instabilities in the non linear regime. Such studies are presently unavailable. However, some insights have been obtained by calculations that treat the blobs as point masses that move in the gravitational potential of the binary where the effects of the magnetic field are included as a resistive force ([15], [36]). 


\subsection{The Accretion Shock at the White Dwarf Surface}

The material that couples on to field lines free falls towards to white dwarf surface forming a strong shock this time at the white dwarf surface with a temperature

$$
T_{\mathrm{s}}^{\mathrm{wd}}=3.7 \times 10^{8}\left(\hat{M}_{1} / R_{\mathrm{wd}, 9}\right) K
$$

The shock cools through cyclotron emission and thermal bremmstrahlung with the former dominating in the higher field / low specific accretion rate regime and the latter dominating in the lower field/higher specific accretion rate regime. The accretion shocks in polars are thus expected to emit hard X-rays in the $20-40 \mathrm{keV}$ band and cyclotron emission in the optical- IR band as primary radiation. Resolvable cyclotron emission lines are often seen in the optical- near IR region which enable a direct measurement of the magnetic field at the accretion shock [29]. The strong angular dependence of the intensity and polarisation properties of cyclotron emission have enabled a very detailed picture to be constructed of the geometry and field distribution in the accretion shocks that form on the white dwarf surface [34]. At the same time, phase dependent emission line studies have enabled the structure of the coupling region to be studied. The major findings are

- The shocks are linearly extended on the white dwarf surface (e.g. see [8], [26] and studies of Stokes imaging by [24]). Typically there are two such regions ("poles") which to a first approximation occur close to the foot points of closed field lines but offset in magnetic longitude. This suggests that the basic underlying field structure is dipolar at least in the coupling region. The linear extension in magnetic longitude $\left(\Delta \phi \sim 50^{\circ}\right)$ suggests by back projection that coupling in the orbital plane occurs over a similarly extended region in rotational longitude. The extension of the shock in magnetic latitude $\left(\Delta \theta \sim 20^{\circ}\right)$ indicates that the radial thickness of the coupling region in the orbital plane is $\Delta R_{\text {coup }} \sim 0.1 R_{\text {coup }}$ with $R_{\text {coup }} \sim R_{\mu}^{\text {st }}[9]$.

- The field strengths at the two poles could differ by a factor $\sim 2$ or more. In the simplest model the field is described by a dipole with its centre offset along the dipole axis (Figure 2). In most systems the dipole axis is observed to point ahead of the line of centres with the weaker magnetic pole pointing towards the companion star (see section 2.4).

- Polars exhibit different states of activity related to changes in the mass transfer rate from the secondary star that could occur on time scales of months to years. There are states when the system is totally inactive or when only pole is active or when both poles are active. A common observation is that only one pole is active when the mass transfer rate is low, while both poles become active as the mass transfer rate increases (e.g. [26]) and the stream penetrates deeper on to the magnetosphere. For the favoured phase locked configuration, the stream first encounters the field lines from the weaker pole which also becomes the stronger accretor.

- The observations also show there is a clear tendency for the material to find its way to the pole that is nearest to the orbital plane as the stream penetrates the magnetosphere. This is also the dynamically favoured pole in the sense that coupled material could pass through the sonic point and free fall to the white dwarf surface without the necessity for additional thermal assistance (see section 2.1). However, additional heating perhaps due to the dissipation of small scale magnetic fields at coupling may allow accretion to occur on to a given pole over a wider azimuthal range from the orbital plane.

- The emission lines (Doppler tomography) show direct evidence of the ballistic stream and a component due to field channelled flow as it leaves the orbital plane (e.g. [24]). The field aligned channeled flow is expected to be accompanied by an azimuthal twist in field lines that is largest at coupling. There is some evidence for such a twist from polarimetric studies of Zeeman spit line emission originating from this region [10]. 
Physics at the Magnetospheric Boundary

\subsection{The soft-X-ray puzzle and the blobby accretion model}

The strongest evidence in support of the blobby accretion scenario has come from X-ray observations of polars.

According to the standard shock model the accretion Luminosity $L_{\mathrm{acc}}=G M \dot{M} / R=L_{\mathrm{br}}+L_{\mathrm{cyc}}$ where $L_{\mathrm{br}}$ and $L_{\mathrm{cyc}}$ are the hard X-ray and cyclotron luminosities emitted by the shock. The shock that typically stands above the stellar surface has a height that is much smaller than the stellar radius so we expect about half of the accretion luminosity to be emitted outwards and the other half to be emitted inwards and be either absorbed and reradiated or to be scattered by the atmosphere. If we assume an efficiency factor $\alpha$ for absorption and thermalisation of hard X-rays the expected ratio of the observed black body to bremmstrahlung (hard-X-ray) fluxes is

$$
\frac{f_{\mathrm{bb}}}{f_{\mathrm{br}}}=\frac{1-\alpha}{1+\alpha}+\frac{1}{1+\alpha} \frac{L_{\mathrm{cyc}}}{L_{\mathrm{br}}}
$$

For most polars with moderate fields the shocks are bremmstrahlung dominated with $L_{\mathrm{br}}>L_{\mathrm{cyc}}$ and we therefore expect $f_{\mathrm{bb}} / f_{\mathrm{br}} \sim 0.5$ (taking $\alpha \sim 0.35$ ). However early observations of several such systems including the prototype AM Her itself indicated $f_{\mathrm{bb}} / f_{\mathrm{br}} \sim 1-100$. It is now well established that a soft-X-ray anomaly is clearly present in a subset of the polars.

The currently accepted explanation was first proposed by Kuijpers and Pringle [17] and came to be known as the "blobby" accretion model. Here it is envisaged that the funnelled accretion flow is inhomogeneous, occurring in the form of blobs in which the specific accretion rate is higher than in a surrounding lower density flow. For a specific accretion rate above a certain limit the ram pressure $P_{\text {acc }}=\rho v_{\mathrm{ff}}^{2}$ of the infalling gas exceeds the photospheric pressure $P_{\mathrm{ph}}$ at optical depth $\sim 1$ in the undisturbed white dwarf atmosphere. The shock is then buried and the energy that is released is reprocessed by the atmosphere and emitted thermally as a black body component at temperatures very much greater the effective temperature of the white dwarf. The critical specific accretion rate $\dot{m}=\rho v_{\mathrm{ff}}$ above which the shock will be buried can be estimated from $P_{\mathrm{ph}}$ to be $\sim 10^{2} \mathrm{gcm}^{-2}$ [17]. Depending on how blobby the flow is, some or all of the accretion energy may be radiated as a black body component.

The clumps that originate during the stream -magnetosphere interaction due to instabilities as discussed in section 2 may be expected to have linear sizes of $(.01-.1) D_{\mathrm{s}}$ but these are likely to have been shredded into smaller blobs before they couple on to field lines. A blob of linear size $l_{\text {coup }}^{\text {blob }}$ along the field and cross-sectional area $A_{\text {coup }}^{\text {blob }}$ perpendicular to the field at coupling will be tidally stretched to $l_{\mathrm{wd}}^{\text {blob }}=l_{\text {coup }}^{\text {blob }}\left(R_{\text {coup }} / R_{\mathrm{wd}}\right)^{1 / 2}$ and compressed to $A_{\mathrm{wd}}^{\text {blob }}=A_{\text {coup }}^{\text {blob }}\left(R_{\text {coup }} / R_{\mathrm{wd}}\right)^{-3}$ by the converging dipolar field lines at the white dwarf surface. The density will be enhanced to $\rho_{\mathrm{wd}}^{\text {blob }}=\rho_{\mathrm{coup}}^{\text {blob }}\left(R_{\mathrm{coup}} / R_{\mathrm{wd}}\right)^{-2.5}$. We may expect time variability of the black body flux on the time scale of entry of the blobs into the photosphere

$$
\Delta t=l_{\mathrm{wd}}^{\mathrm{blob}} / v_{\mathrm{ff}}=620\left(l_{\text {coup }}^{\text {blob }} / R_{\text {coup }}\right)\left(R_{\text {coup }, 10}\right)^{3 / 2} \hat{M}_{1}^{-1 / 2} R_{\mathrm{wd}, 9}^{1 / 2} \mathrm{~s}
$$

that is uncorrelated with the hard-Xray emission. Observations of cyclotron emission regions suggest that $\Delta R_{\text {coup }} / R_{\text {coup }} \sim 0.1$ with $R_{\text {coup }} \sim R_{\mu}^{\text {st }}$ (see section 3.2) and if we assume that $l_{\text {coup }}^{\text {blob }}<\Delta R_{\text {coup }}$ we may expect to detect variability on time scales of of about a hundred seconds or less. The total energy released by a blob $\epsilon^{\text {blob }}$ is related to its mass from

$$
m^{\text {blob }}=8 \times 10^{16} \epsilon_{34}^{\text {blob }} \hat{M}_{1}^{-1 / 2} R_{\mathrm{wd}, 9}^{1 / 2} \mathrm{~g}
$$

V1309 Orion is a polar that shows unequivocal evidence for blobby accretion[27]. The system has a bright phase dominated by soft X-ray emission $\left(k T_{\mathrm{bb}}=45 \mathrm{eV}\right)$ with $f_{\mathrm{bb}} / f_{\mathrm{br}} \geq 6700$. The emission 


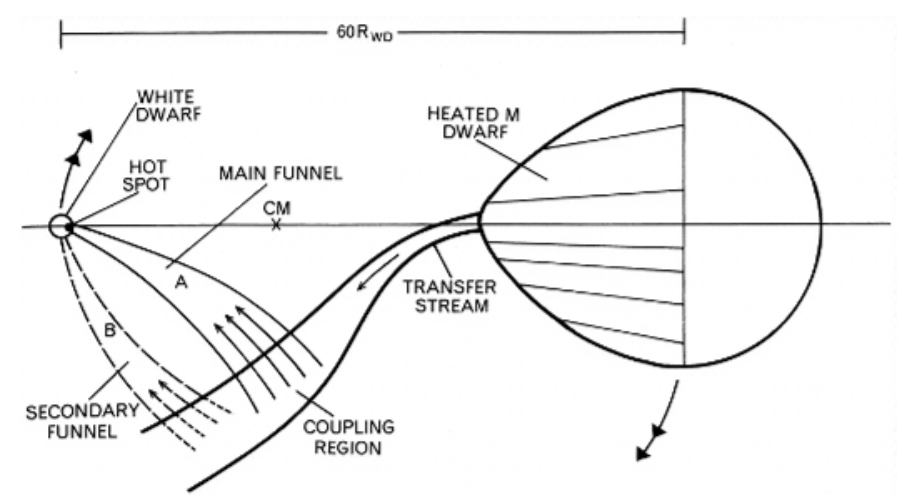

Figure 2. The two pole model (schematic) for a field distribution that is modelled by a dipole that is off set from the centre along the dipole axis. The magneto-static coupling is such that the magnetic axis leads the line of centres with the weaker pole pointing towards the secondary star. As the stream penetrates the magnetosphere it preferentially feeds the dynamically favoured pole - that is the pole closest to the orbital plane. First the weaker field main pole and then the stronger field second pole further downstream.

occurs mostly in time resolved flares that typically last for $10 \mathrm{~s}$ and emit a total energy of $\epsilon=10^{34} \mathrm{erg}$. The simple model of blob entry into the photosphere discussed above suggests a typical blob mass of $2 \times 10^{18} \mathrm{~g}$ for this system and a specific accretion rate $\dot{m}>300 \mathrm{gcm}^{-2}$ well above the value at which the ram pressure of the flow will bury the shock. If the density at coupling $\mathrm{s}$ that in the cooled immediate post shock region $\left(\rho_{3}\right)$ the implied blob size is $\sim 2 \times 10^{7} \mathrm{~cm}$. The presence of a background hard X-ray component seen at all phases unrelated to the bright phase shock emission that can be attributed to the shock at the stream-magnetosphere interaction also supports this model [27].

The degree of clumpiness of the funnel flow will ultimately depend on the nature of the instabilities in the coupling region. These will depend on binary parameters, field structure, and the orientation of field lines relative to the stream. V1309 Orion has a polar field of $61 \mathrm{MG}$ which is about a factor 2 higher than the average field for a polar but also has an unusually long orbital period. There is some observational evidence that the higher field systems are more likely to show a soft - X-ray anomaly ([25], [2]). This could be because the lower limit to the length scale of the blobs that first emerge from the region of the stream-magnetosphere interaction is set by the strength of the magnetic field as indicated by theory. In the case of the IPs where coupling occurs from a disc and similar instabilities are expected there is no evidence for clumpy accretion at least on the time scales seen in the polars.

\section{Conclusions}

The study of polars have brought to the fore the importance of MHD and other instabilities that occur at the magnetospheric boundary in shaping the nature of the field channelled flow. Such instabilities 


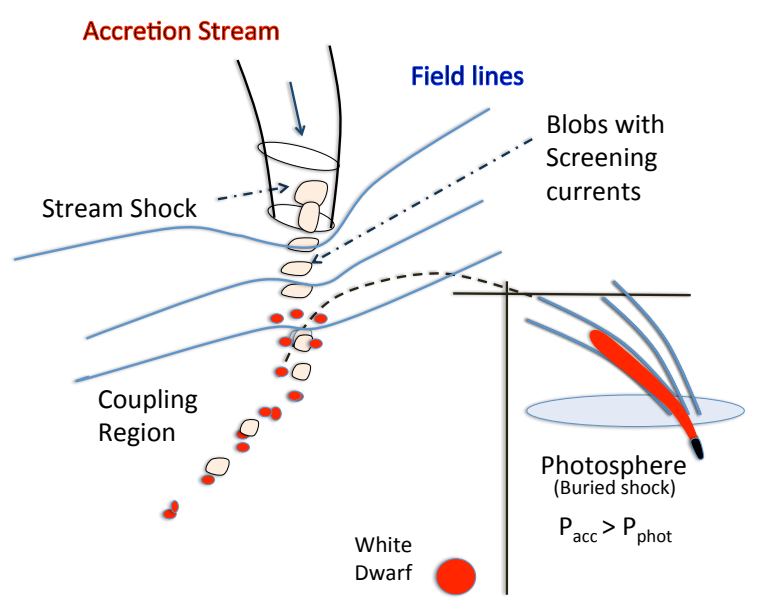

Figure 3. The strong stream shock hypothesis (Scenario 1). Here the stream encounters a shock at the stream magnetospheric radius at which the gas fragments into clumps of various masses due to the Magnetic Rayleigh Taylor instability limited by the strength of the magnetic field. The larger masses initially follow a dynamical trajectory resisted by the magnetic field but are then stripped into smaller masses by the Kelvin-Helmholtz instability that then diffuse in to field lines. The blobs are stretched into discrete filaments as they free fall along field lines towards the white dwarf surface. If the specific accretion rate is in excess of $\sim 10^{2} \mathrm{gcm}^{-2}$ the resulting shock is buried in the white dwarf atmosphere and the accretion energy is released as a reprocessed soft-X-ray black-body component.

are usually not encapsulated in the large scale MHD simulations of accretion flows on to magnetic stars.

There are two possible closely related scenarios that have been advanced to explain the observations of polars. In the first scenario a strong shock forms at the stream- magnetosphere boundary. Various interchange instabilities such as the magnetic Rayleigh-Taylor instability in the cooled post shock region results in the steam fragmenting into clumps of gas with length scales and masses that are set by the strength of the field. As this material penetrates the magnetosphere the initially less massive clumps couple on to field lines and feed the main pole (the stronger accretor) while the more massive clumps penetrate further down stream and are shredded into smaller blobs by the Kelvin-Helmholtz instability which then couple on to field lines and feed the second pole (the weaker accretor) (see Figure 3). This model is consistent with observations of systems such as V1309 Orion where there is direct evidence for X-ray emission at $\sim 0.5 \mathrm{keV}$ from a strong stream shock but perhaps not for other systems where evidence for such emission has not been reported.

In an alternative but closely related scenario also discussed in the literature the stream is continuously decelerated by a series of weak shocks as it plunges into the magnetosphere. MHD and other instabilities at its surface shred the stream into small enough mass components that diffuse into corotating field lines and deplete the mass of the stream until it is exhausted. This scenario is similar to what may be in operation in the inner transition region in an accretion disc where material is similarly depleted from its surface layers until the disc material is exhausted. However to date there has been no reported evidence for blobby accretion in the IPs. 


\section{References}

[1] Arons, J. \& Lea, S. M., Ap. J. 207, 914-936 (1976)

[2] Beuermann, K. \& Schwope, A. D., in Interacting Binaries Stars, ASP Conf. Ser. 56, 119-122 (1994)

[3] Byung-Il, J., Norman, M. L. \& Stone, J. M., Ap. J. 453, 332-349 (1995)

[4] Campbell, C. G., MNRAS, 219, 589-596 (1986)

[5] Campbell, C. G., MNRAS, 403, 1339-1352 (2010)

[6] Campbell, C. G., MNRAS, 409, 433-446 (2010)

[7] Campbell, C. G., MNRAS, 414, 3071-3083 (2011)

[8] Ferrario, L.\& Wickramasinghe, D. T., Ap.J, 357, 582-590 (1990)

[9] Ferrario, L.\& Wickramasinghe, D. T.\& Tuohy, I. R., Ap.J, 341, 327-339 (1989)

[10] Ferrario, L., Wickramasinghe, D. T. \& Schmidt, G., MNRAS, 331, 736-744 (2002)

[11] Ghosh, P. \& Lamb, F. K., Ap. J. 232, 259-276 (1979)

[12] Hameury, J. M., King, A. R. \& Lasota, J. P., MNRAS, 218, 695-710 (1986)

[13] Hellier, C. Star-Disc Interaction in Young Stars, IAU Symposium 243, Eds: Bouvier, J. EAAppenzeller, I. (2007)

[14] Hessman, F. V., Ap. J., 510, 867-873 (1999)

[15] King, A. R., MNRAS, 261, 144-148 (1993)

[16] King, A. R. \& Lasota, J., Ap. J. 378, 674-681 (1991)

[17] Kuijpers, J. \& Pringle, J. P., A\&A 114, L4-L6 (1982)

[18] Lamb, D., Q..\& Melia, F., Ap\&SS. 131, 517-547 (1987)

[19] Li, J., Wickramasinghe, D. T. \& Rudiger, G., Ap. J.,469, 765-775 (1996)

[20] Li, J., Wu, K. \& Wickramasinghe, D. T., 270, 769- 773(1994)

[21] Li, J., Wu, K. \& Wickramasinghe, D. T., 286, L25-L28 (1997)

[22] Lubow, S. H. \& Shu, F. H., Ap. J. 198, 385-405 (1975)

[23] Norton, A. J., Wynn, G. A. \& Somerscales, R. V., Ap. J. 614, 349-357 (2004)

[24] Potter, S. B., Romero-Colmenero, E., Watson, C. A., Buckley, D. A. H. \& Phillips, A., MNRAS, 348, 316-324 (2004)

[25] Ramsey, G., Mason, K., Cropper, M.,Watson, M.G.\&Clayton, K. L., MNRAS, 270, 692-696 (1994)

[26] Romero-Colmenero, E., Potter, S. B., Buckley, D. A. H., Barett, P. E. \& Vrielmann, S., MNRAS, 339, 685-769 (2003)

[27] Schwarz, R., Reisch, K., Beuermann, K. \& Burwitz, V., A\&A 442, 271-279 (2005)

[28] Tout, C. A, Wickramasinghe, D. T.,Liebert, J. , Ferrario, L. \& Pringle, J. E., MNRAS, 387, 897-901 (2008)

[29] Visvanathan, N. \& Wickramasinghe, D. T., Nature, 281, 47-48 (1979)

[30] Wang, Y. -M.\& Nepveu, M., Astron\&Astrophys. 118, 267-274 (1983)

[31] Warner, BCataclysmic Variable Stars (Cambridge University Press, 1995)

[32] Warner, B. \&Wickramasinghe, D. T., MNRAS, 248, 370- 376 (1991)

[33] Wickramasinghe, D. T. \&Wu, K., MNRAS., 253, 11P-15P (1991)

[34] Wickramasinghe, D. T. \&Ferrario, L., PASP.112, 873- 924 (2000)

[35] Wickramasinghe, D. T. , Tout, C. \&Ferrario, L., MNRAS submitted (2013)

[36] Wynn, G. \& KIng, A. R., MNRAS 275, 9-21( 1995)

[37] Wynn, G., KIng, A.R. \& Horne, K., MNRAS 280, 436-446( 1997) 